\title{
Produção de Redes e o público-privado: implicações no cuidar da doença respiratória crônica
}

\author{
Network production and the public-private: implications in caring for chronic respiratory disease \\ Producción de redes y público privado: implicaciones para el cuidado de las enfermedades \\ respiratorias crónicas
}

\author{
Márcio Costa de Souza \\ ORCID: https://orcid.org/0000-0002-4922-6786 \\ Universidade do Estado da Bahia, Brasil \\ E-mail: mcsouzafisio@gmail.com \\ Kamila Freitas Trindade \\ ORCID: https://orcid.org/0000-0003-1603-8196 \\ Universidade do Estado da Bahia, Brasil \\ E-mail: freitastmila@gmail.com \\ Rafaela Silva Santos \\ ORCID: https://orcid.org/0000-0002-7755-7734 \\ Universidade do Estado da Bahia, Brasil \\ E-mail: r.santoss1507@gmail.com \\ Vitória Cézar Santos Gonçalves Brito \\ ORCID: https://orcid.org/0000-0003-0578-3019 \\ Universidade do Estado da Bahia, Brasil \\ E-mail: vi.cezaar4@gmail.com \\ Juliana Costa dos Santos Borges \\ ORCID: https://orcid.org/0000-0002-5711-8136 \\ Universidade do Estado da Bahia, Brasil \\ E-mail: jucsborges@gmail.com \\ Jairrose Nascimento Souza \\ ORCID: https://orcid.org/0000-0002-7044-3371 \\ Universidade do Estado da Bahia, Brasil \\ E-mail: rnfisio10@gmail.com \\ Magno Conceição das Mercês \\ ORCID: https://orcid.org/0000-0003-3493-8606 \\ Universidade do Estado da Bahia, Brasil \\ E-mail: mmerces@uneb.br \\ Roberto Rodrigues Bandeirqa Tosta Maciel \\ ORCID: https://orcid.org/0000-0002-4912-6005 \\ Universidade do Estado da Bahia, Brasil \\ E-mail: rmaciel@uneb.br \\ Fernanda Warken Rosa Camelier \\ ORCID: https://orcid.org/0000-0003-2540-0142 \\ Universidade do Estado da Bahia, Brasil \\ E-mail: fcamelier@uneb.br \\ Aquiles Assunção Camelier \\ ORCID: https://orcid.org/0000-0001-5410-5180 \\ Universidade do Estado da Bahia, Brasil \\ E-mail: aquilescamelier@yahoo.com.br
}

\begin{abstract}
Resumo
Objetivo: analisar a produção de redes e a interface com o público-privado no cuidado de pessoas com doenças respiratórias crônicas. Metodologia: utilizou a abordagem qualitativa exploratória, no qual os dados foram coletados por meio de um roteiro semiestruturado. Os locais escolhidos para a coleta de dados foram as Unidades de Saúde da Família que se encontram localizadas em um Distrito Sanitário de Salvador-Bahia. A amostra é composta por 15 participantes escolhidos pela saturação das respostas. Resultados: As redes de cuidado também são construídas pelo usuário, constituindo a rede viva e o público-privado faz parte dessa tessitura a partir dos caminhos percorridos pelo o usuário entre os serviços de naturezas diferentes para que possam resolver suas demandas no ato de tecer suas próprias redes, há um déficit de interlocução nos serviços, e o itinerário construído entre público-privado pode ser produzido pelos profissionais de saúde. Considerações finais: As redes de atenção apresentam problemas
\end{abstract}


organizacionais, o que interfere no cuidado integral e a continuidade deste, e ainda que há uma interface entre o público e o privado para atender as necessidades dos usuários.

Palavras-chave: Assistência centrada no paciente; Avaliação de resultados em cuidados de saúde; Continuidade da assistência ao paciente.

\begin{abstract}
Aim: to analyze the networks production and the interface with the public-private sector in the care for people with chronic respiratory diseases. Material and methods: used the qualitative exploratory approach, in which data were collected through guided by a semi-structured script. The locations chosen for data collection were the Family Health Units, which are located in a Sanitary District of Salvador-Bahia. The sample consists of 15 participants chosen by saturation of responses. Results: The care networks are also built by the user, constituting the living texture and the public-private part of this network from the paths taken by the user between services of different natures so that they can solve their demands in the act of weaving their careers. networks themselves, there is a deficit of interlocution in services, and the itinerary built between public-private can be produced by health professionals. Conclusion: The health care networks present organizational problems, which interferes with integral care and its continuity, and that there is an interface between the public and the private to meet the needs of users.
\end{abstract}

Keywords: Patient-centered care; Outcome assessment health care; Continuity of pacient care.

\title{
Resumen
}

Objetivo: analizar la producción de redes y una interfaz con lo público-privado en la atención de personas con enfermedades respiratorias crónicas. Metodología: se utilizó un enfoque cualitativo exploratorio, en el que los datos se recolectaron a través de un guión semiestructurado. Los lugares elegidos para la recolección de datos fueron las Unidades de Salud de la Familia que se caracterizan en un Distrito Sanitario de Salvador-Bahía. La muestra consta de 15 participantes, elegidos por la saturación de respuestas. Resultados: Las redes de atención también son construidas por el usuario, constituyendo una tejido vivo y lo público-privado forma parte de esta red a partir de los caminos que recorre el usuario entre servicios de distinta naturaleza para que pueda resolver sus demandas en el acto de tejiendo sus propias redes, hay un déficit de interlocución en los servicios, y el itinerario construido entre lo público y lo privado puede ser elaborado por los profesionales de la salud. Consideraciones finales: Las redes de atención presentan problemas organizativos, que interfieren con la atención integral y su continuidad, aunque existe una interfaz entre lo público y lo privado para atender las necesidades de los usuarios.

Palabras clave: Atención dirigida al paciente; Evaluación de procesos y resultados en atención de salud; Continuidad de la atención al paciente.

\section{Introdução}

As Redes de Atenção à Saúde (RAS), foram implementadas no âmbito SUS como estruturas organizativas no campo da saúde, no qual estabelece por meio de ações e serviços de saúde com tecnologias do cuidar com diferentes densidades, em que devem estar integradas no âmbito técnico, logístico e da gestão, com a finalidade de prover um cuidado integral e resolutivo (Brasil, 2014). Desse modo, a RAS deve ser organizada e articulada, com a finalidade de reduzir a fragmentação do cuidado, o que é imprescindível para atender as necessidades das pessoas com doenças crônicas (Viana et al, 2018).

Destarte, a rede institucionalizada segue uma lógica burocratizada, que indica aos usuários e aos trabalhadores caminhos esquematizados a partir de diretrizes e protocolos para adentrar na rede (Rosa et al, 2016a). No entanto, observa-se que, frequentemente, os usuários constroem suas próprias redes com diferentes conexões. Essa forma de circulação dos usuários tecendo suas próprias redes de sociabilidade e cuidado caracteriza a rede viva (Merhy et al, 2014), que corresponde a todos os movimentos construídos sejam eles fora dos protocolos, fora das regras, fora da regulação (Rosa et al, 2016b). Eles se apropriam de um conhecimento prático proveniente da própria experimentação e observação de como conseguir o acesso ao cuidado que julgam como necessário (Meneses et al, 2017).

O mix público-privado aparece na construção das redes a partir dos caminhos percorridos pelo o usuário entre o serviço público e privado para ter suas demandas resolvidas. O setor privado apresenta-se de duas formas, como complementar ao SUS, quando é preciso pagar para adquirir serviços que não são oferecidos pelo mesmo e suplementar quando se trata da venda de planos de saúde (Cardoso et al 2017). Assim, o itinerário terapêutico do cuidado produzido pelo o usuário pode ser de 
atravessamentos entre o público e o privado, no qual permite ao usuário acessar serviços particulares, quanto serviços públicos.

Com base nas informações anteriores, objetiva-se com esse estudo, analisar a produção de redes e a interface com o público-privado no do cuidado de pessoas com doenças respiratórias crônicas.

\section{Metodologia}

Optou-se como processo metodológico para a realização da pesquisa, a abordagem qualitativa, de caráter exploratório, o qual permite que o fenômeno observado seja conhecido com uma maior espontaneidade (Tonetto et al., 2014). Esta premissa confere uma maior familiaridade entre o pesquisador e o problema a ser analisado (Gil, 2017).

No que tange ao local de estudo para a realização da pesquisa, a escolha foi de forma intencional, quatro Unidades de Saúde da Família (USF) pertencentes a um Distrito situado no município de Salvador, no Estado da Bahia.

No tocante aos participantes da pesquisa foram escolhidos os usuários acometidos por Doença Respiratórias Crônicas (DRC), familiares e/ou cuidadores e trabalhadores de saúde. Como critérios de inclusão, englobam-se os usuários acometidos por Doença Respiratórias Crônicas (DRC) em acompanhamento USF do Distrito sanitário estudado, familiares e/ou cuidadores e trabalhadores de saúde que atuam na assistência aos pacientes acometidos por DRC. Ainda no que se refere ao grupo "trabalhadores de saúde", se estabeleceu que o mesmo fosse constituído por qualquer pessoa que atue na USF, independente da formação específica para saúde (médicos, enfermeiros, atendentes, e demais trabalhadores do serviço). Como critérios de exclusão, não foram entrevistados os menores de 18 anos, trabalhadores da saúde da instituição referida que estiverem de férias, folga ou licença e usuários e familiares e/ou cuidadores que não desejaram participar da pesquisa.

A ferramenta utilizada para a produção dos dados consiste na aplicação de entrevistas, norteadas por um roteiro semiestruturado, pelo fato deste instrumento permitir liberdade durante a produção dos dados o que contribui para a relação intersubjetiva entre o participante da pesquisa e o pesquisador (Minayo \& Deslandes, 2016). No entanto, o processo de pesquisa qualitativa não admite visões isoladas, ela se desenvolve em interação dinâmica, retroalimentando-se e reformulandose constantemente, revelando assim a posição do pesquisador diante dos fenômenos estudados, o qual passa a ser incluso nesse processo de saber-fazer (Silva et al., 2018). Desta forma, essas ferramentas inicialmente citadas serão utilizadas para dar visibilidade aos acontecimentos nos locais estudados, utilizando-se, portanto, o encontro como potencializador na produção dados, no acontecimento.

Durante as entrevistas foi utilizado um gravador (Motorola XT 311) para registrar com maior detalhamento os depoimentos, no entanto, tal meio só será utilizado mediante prévia autorização do participante. Para a realização da entrevista estruturada a mesma possui um roteiro em que se divide em um tópico com alguns subtópicos: Produção de diagnóstico com o subtópico: Projeto Terapêutico Singular, Pontos de atenção, interdisciplinaridade, Referência/Contra referência, Gestão de casos, coordenação do cuidado e apoio matricial.

Tendo em vista a sistematização das informações coletadas para uma posterior análise, foram realizados os seguintes passos: inicialmente ordenou-se as informações por meio de um mapeamento de todos os dados obtidos (transcrição de gravações, releitura de material, organização de gravações, dos dados do diário de campo). Por conseguinte, realizou-se a classificação das informações, a partir da identificação dos núcleos de sentidos e por conseguinte elaborou-se as chamadas categorias empíricas. Nesta pesquisa, após o devido agrupamento e análise dos dados, encontrou-se as categorias empíricas: "Produção de redes" e "relação público-privado".

Por fim, para o momento de análise final das informações, houve a articulação dos dados empíricos com os referenciais teóricos da pesquisa e documentos, promovendo assim, a relação entre o concreto e o abstrato, o geral e o 
particular, a teoria e a prática. Ao formular analisadores devemos cruzar as informações obtidas nas diferentes ferramentas de pesquisas.

Esta pesquisa foi aprovada no CEP da Universidade do Estado da Bahia sob o no do CAAE: 61478016.0.0000.0057, baseado na Resolução 466/12 do Conselho Nacional de saúde sobre as Diretrizes e Normas Regulamentadoras de Pesquisa envolvendo seres humanos.

\section{Resultados e Discussão}

Ao final do processo de coleta de dados, contabilizou-se 15 entrevistados. Em pesquisas de caráter qualitativo, que utilizam entrevistas como ferramenta para coleta de dados, a saturação é verificada quando os dados, após a devida análise, apresentam consistência em qualidade e densidade quantitativa, ou seja, ausência de elementos novos dados no material tratado (Fusch \& Ness, 2015). Dessa forma, o quantitativo total de 15 entrevistas apresentou-se suficiente em decorrência do não aparecimento de novas informações gerando assim, uma repetição de dados.

\subsection{Produção de Redes}

As Redes de Atenção à Saúde (RAS) foram implementadas no Sistema Único de Saúde (SUS) com o intuito de garantir a integralidade do cuidado (Brasil, 2014). Desse modo, a rede, no campo da saúde, refere-se a uma compreensão acerca da organização dos serviços de saúde e suas conexões, bem como a articulação de pessoas ou ações em saúde. Assim, os elementos fixos da rede podem ser pessoas e/ou serviços, que podem ser organizadas de forma individual, como trabalhadores ou usuários, ou organizadas em conjuntos, como equipes (Amaral \& Bosi, 2017).

Para Deleuze (2002), os discursos e práticas tentam entender as redes como um conceito representação, retirando desta o que produz os seus sentidos: os sujeitos nas suas experiências vividas. Frequentemente, o mundo da rede de cuidados, gira em torno das suas próprias lógicas de saberes, o que coloca usuário como seu objeto de ação, como alguém desprovido de conhecimentos e experiências. No entanto, os usuários constroem suas próprias redes com diferentes conexões, pois eles são redes vivas de si próprios e estão o tempo inteiro produzindo movimentos, elaborando saberes, construindo e partilhando cuidados (Merhy et al, 2014).

Diante disso, pode-se notar um exemplo de como usuários tecem suas próprias redes, visto que, muitas vezes, algumas ofertas de determinado serviço de saúde são disseminadas a partir do compartilhamento de alguma vivência do usuário, conforme a fala descrita abaixo.

Aí ela conversando comigo me disse que lá no hospital tinha um setor de fibrose que eu nem sabia. Ela disse que nesse setor tem 5 farmácias e ninguém passou isso para mim porque se tivessem me falado antes, eu já ia levar os papéis dele de remédio de alto custo e levava para lá. (Entrevistado 4).

Nós pedimos para que as pessoas divulguem, entendeu? A gente divulga tanto entre a gente mesmo, por exemplo, se eu tenho uma vizinha que fuma eu posso convidá-la para participar do grupo. Ou seja, geralmente o grupo é passado de boca a boca, vai sendo divulgado e muitas pessoas aparecem. (Entrevistado 1).

Essas falas revelam como as redes são vivas e se produzem, apresentam de forma não analógicas, montam e desmontam, sem que exista um controle sobre elas. Diferente da rede formal, que possui as estruturas e os procedimentos estabelecidos para a função cuidadora, a rede informal não é previamente estabelecida para isso, mas exerce esse papel ao participar das diferentes situações do cuidado. Desse modo, são traçados linhas e conexões entre usuários, profissionais, 
equipamentos e territórios que fogem àquelas estabelecidas na rede institucionalizada pelo sistema de saúde (Maximino et al, 2017).

Outro ponto importante para a construção das RAS é a relação existente entre os serviços de saúde. A rede construída entre os profissionais de um serviço de saúde é imprescindível para a sustentação do cuidado no cotidiano, bem como a relação estabelecida entre os diferentes níveis de atenção. Assim, os procedimentos de articulação como encaminhamentos, contato telefônico e visitas institucionais são artifícios que possibilitam o cuidado compartilhado e uma efetiva construção da rede (Bermudez \& Batista, 2017).

As impressões compartilhadas pelos usuários denotam a falta de interlocução da rede, a falha na comunicação entre os serviços e como a responsabilidade do cuidado é transferida aos usuários, como pode ser visto nas entrevistas abaixo.

Ela (assistente social - grifo nosso) me deu os papéis e disse que era melhor eu correr atrás. Eu disse "é brincadeira isso" [...]. Eu que fui atrás porque disseram no hospital que iam encaminhar, né. Mas eu que fui atrás mais de $5 x$. (Entrevistado 4).

Ele (médico- grifo nosso) me passou o raio x e mandou eu voltar, eu voltei, ele deu uma olhada no raio x e disse assim: "Não se preocupe muito não, mas veja se você consegue marcar um pneumologista". Aí eu vim aqui e consegui marcar para hoje. Eu vim aqui. No mesmo dia que eu fui atendido lá, ele mandou eu vim marcar aqui. (Entrevistado 9).

A partir do que foi compartilhado, é possível perceber, que algumas vezes, a responsabilidade de chegar a determinado serviço é transferido ao usuário, cabe ao usuário(a) buscar o que é necessário para a continuidade do cuidado. Isso aponta para uma capacidade reduzida de conectividade da rede, o que gera ausência de movimento, que pode se caracterizar na capacidade de produzir relações ou até mesmo de ruptura, ou seja, a fluidez existente nas relações podem ser potencializadas positivamente ou negativamente (Amaral \& Bosi, 2017).

Desta forma, o funcionamento adequado da rede requer ainda que a relação predominante entre os serviços de saúde, construída por encontros potentes e com saberes diferentes, no qual estabeleça relações de poder mais horizontalizadas, com o intuito de superar as fragmentações existentes e produzir uma interlocução entre esses serviços para garantir um cuidado integral (Aoki et al, 2017).

Assim, um ponto que tem potencial de ser um dispositivo articulador da rede é o matricialmente, que é um arranjo organizacional e uma metodologia para a gestão do trabalho em saúde que tem a capacidade de estimular a produção de novos padrões de relacionamento entre equipe e amplia o compromisso dos profissionais com o cuidado em saúde, isto faz com que barreiras organizacionais que dificultam a comunicação sejam superadas (Rocha et al 2016; Peduzzi et al, 2020). A partir desta lógica, há indícios de facilitação para a construção de novos processos de trabalho colaborativos, com ações pautadas na interprofissionalidade, espaços coletivos com troca de experiências, construção horizontal de saberes, portanto, encontros que viabilizam a produção do cuidado (Agreli et al., 2016).

Quando um único serviço de saúde não é capaz de resolver todas as demandas a ele direcionadas, é preciso deslocar o usuário para outro serviço apropriado. Para que esse fluxo ocorra com eficiência é preciso conhecer as condições de acessibilidade da unidade de saúde referenciada e as chances de prática interprofissional colaborativa, no qual busca antecipar adequadamente a eficácia ou impossibilidade de concretização da resolutividade do cuidado (Amaral \& Bosi, 2017; Quintana, 2020). Desse modo, é necessária a existência de comunicação fluida e efetiva entre os serviços de saúde para dar continuidade à atenção. 
Desta forma, é de fundamental importância que tenham políticas que organizem a comunicação da equipe e entre os serviços de saúde, e assim possam construir ações na rede de atenção que estabeleçam a produção de vínculos, uma simetria de diálogo entre profissional-usuário, desta forma possa compreender o cuidar como um processo de formação permanente, e consequentemente centrada no do usuário (Agreli et al. 2016; Souza et al, 2017; Seixas et al., 2019). Quando tais falhas predominam e comprometem o fluxo do usuário pelo sistema, eles acabam buscando e criando maneiras de acessar os serviços de saúde, criando fluxos que respondam às suas demandas reais ou simbólicas (Viana et al, 2018).

Para que exista integração entre as redes de saúde é necessário construir um efetivo sistema de referência e de contrarreferência. No qual, a referência corresponde ao encaminhamento do usuário para serviços especializados de acordo com a complexidade do seu caso, enquanto a contrarreferência refere-se ao retorno do usuário ao serviço inicial. Porém, alguns fatores interferem no sistema de referência e de contrarreferência. Dentre eles, temos limitada oferta de consultas e exames, má organização das atividades de regulação e inexistência ou precariedade da contrarreferência (Costa et al, 2015).

Os relatos a seguir corroboram com a precariedade ou falta de contrarreferência predominante nos serviços de saúde.

Basicamente, quando precisa de uma especialidade, o médico faz uma requisição solicitando e a gente encaminha o paciente, faz uma ficha de referência se necessário, mas desse tempo que eu estou aqui nunca vi uma contra referência. O paciente sai e a gente fica com aquela sensação "será que vai voltar?". [...] Então, minha experiência sobre as redes é essa de ter esse sentimento de mandar o paciente e não ter esse retorno. O paciente sai e a gente não sabe se de lá ele foi para outro lugar ou se ficou bom. Eu nunca vi um caso de um paciente voltar para cá e dá um retorno a gente ou voltar a ficha da contrarreferência, eu nunca vi. Então a gente tem muito esse sentimento de perda. (Entrevistado 1).

Alguns, sim. Eles não voltam, exatamente, para me dá o retorno; mas, quando eles voltam para uma consulta eu pergunto "foi ao otorrino?”. Porque como é Saúde da Família tem esse vínculo. (Entrevistado 3).

Dessa forma, nota-se que a falta de resposta para o serviço inicial compromete a continuidade do cuidado, despertando a sensação de perda do usuário na equipe de saúde, além de acarretar novos encaminhamentos, muitas vezes, desnecessários, pois o problema já pode ter sido sanado. A contrarreferência é relevante na articulação da rede quando exercida de forma coerente e efetiva, com acompanhamento e devolução do caso, o que produz uma comunicação entre os serviços, e assim estabelecendo de fato uma rede entre as estruturas existentes (Merhy, 2012). Esse tipo de ação, preserva os princípios da integralidade e resolutividade.

\subsection{Relação público-privado}

Os serviços privados aparecem na rede de cuidados como complementares à organização do aparato público do SUS, quando o sistema não tem capacidade de oferta de certos tipos de procedimentos, então é preciso comprar serviços, e ainda pode ser utilizado de forma; suplementar quando se trata da venda de planos de saúde (Meneses et al, 2017).

Essas dimensões do público-privado se fazem presente na construção das redes de cuidado dos entrevistados, como pode ser visto na fala a seguir.

Eu tenho plano de saúde, mas não tirei não. Apesar do plano, quem cuida deles é Dra X aqui (na USF). [...] E hoje tanto faz o SUS como o plano de saúde estão a mesma coisa. (Entrevistado 2). 
Observa-se que mesmo tendo acesso ao setor privado, o itinerário do cuidado exercido por essa usuária perpassa pelo serviço público, sendo equiparado com os serviços ofertados pela rede particular. Nesta realidade experenciada, o setor privado funciona como suplementar, existindo uma clientela de consumidores específica, a de consumidores do produto, denominado de seguro de saúde (Merhy, 2012).

É importante destacar que essa entrevistada é uma agente comunitária de saúde e que esses profissionais fazem um potente elo entre as redes formais e informais. Eles são profissionais do serviço, mas também familiares, amigos e vizinhos dos usuários, detendo informações e realizando ações que articulam o público e o privado (Maximino et al, 2017). Nota-se também, que enquanto trabalhadora da saúde, ela sabe como acessar os serviços e criar uma rede de cuidados que se intercala entre o público e o privado, aumentando, dessa forma, a resolubilidade das suas demandas.

Outro ponto observado é como o itinerário público-privado pode ser produzido por determinados profissionais, como pode ser visto na fala abaixo.

Meu filho era pelo plano que eu tinha. Quando o otorrino foi para o HapVida, ele me disse "qualquer coisa me procure lá (no Santa Izabel) ou na Faculdade de Medicina" porque ele trabalha lá e tem uma clínica particular. Então qualquer coisa ele me diz "não fica trazendo aqui não que é muita agonia (Santa Izabel), muita gente". Então, meus filhos acabam se expondo muito. (Entrevistado 2).

Percebe-se na fala acima que o profissional de saúde propõe a usuária que caminhe tanto em unidades de saúde privada quanto públicas, norteada pela agenda do mesmo. Vale a pena destacar que, a atenção em saúde deve ser centrada na figura do usuário, para atender as necessidades deste, no entanto, o que ocorre é um fluxo estabelecido que atenda a necessidade do profissional (Agreli et al., 2016). Assim, a realidade do cuidado, neste caso, é estabelecida por andanças e encaminhamentos entre os serviços público e privado, e que, por vezes, pode furar a organização de fluxos e processos burocráticos, além da desconstrução das linhas de cuidado de diversas condições de saúde (Rosa et al, 2016a). Por outro lado, o trabalho dos profissionais no público e no privado pode ser sinérgico na atenção das necessidades do usuário, potencializando a qualidade da atenção, fazendo por si só uma interface público-privado (Meneses et al, 2017).

Apesar de muitos indivíduos ainda ver a saúde como algo a ser consumido, imaginário simbólico construído pelo usuário-consumidor, há quem enxergue a saúde como um direito social, imaginário simbólico do usuário-cidadão (Rosa et al, 2016). As falas a seguir trazem, mesmo que de forma inconsciente, a perspectiva do usuário-cidadão.

A minha menina trabalha lá e a gente ia pagar, mas é muito cara uma consulta com o pneumologista, então, eu disse que não ia pagar porque ia pelo SUS. Não levou oito dias e eu consegui marcar com o médico. [...] Na época Dr. Z passou, só uma vez, sem pagar. Tudo pelo SUS, porque ele é maravilhoso. Meu plano de saúde é o SUS. (Entrevistado 5).

A participação popular é imprescindível para alcançar a qualidade nos serviços e ações de saúde, pois o coletivo é capaz de identificar os problemas e as soluções relativas às suas demandas (Hoppe et al, 2017). Uma vez que a criação do SUS foi um direito social conquistado pela população, é preciso cada vez mais criar consciência de direitos e deveres nos usuários do SUS, para que eles estejam engajados na luta pela defesa do SUS e sejam atores sociais e não apenas receptores do cuidado. 


\section{Considerações Finais}

Diante da realidade vivenciada, infere-se que as RAS ainda possuem problemas organizacionais, como falta de interlocução entre os serviços e déficit de comunicação, comprometendo a integralidade e a continuidade do cuidado dos usuários. Diante disso, o usuário, em busca da resolução das suas demandas, constrói sua própria rede, traçando um itinerário do cuidado que, por vezes, sai do que é sistematizado.

O caminho percorrido pelo usuário não é destituído de um saber e lógica, pois ele é um ser provido de conhecimento, experiências e observação e os utiliza para conseguir acesso ao cuidado necessário. Nesse itinerário, os usuários podem acessar serviços públicos e privados na tentativa de amplificar as possibilidades de resolução da sua demanda. Assim, as formas utilizadas para solucionar seus problemas de saúde apontam para a construção de uma rede que é viva, monta e desmonta de acordo com a necessidade de cada um. Estudos que complementem, descrevam as Redes de Atenção em Saúde e as alternativas vinculadas a elas são cada vez mais necessários para promoção de um cuidado efetivo e de fato centrado no paciente sobretudo no contexto das afecções crônicas.

\section{Referências}

Agreli, H. F., Peduzzi, M. \& Silva, M. C. (2016). Atenção centrada no paciente na prática interprofissional colaborativa. Interface - Comunicação, saúde e educação, 20(59), 905-916.

Amaral, C. E. M. \& Bosi, M. L. M. (2017). O desafio da análise de redes de saúde no campo da saúde coletiva. Saúde e Sociedade, 26(2), 424-434.

Aoki, M. et al. (2017). Challenges on network care considering the perceptions of preceptors of a Pet-Network regarding people with disabilities and at-risk infants: access, comprehensiveness and communication. Cadernos Brasileiros de Terapia Ocupacional, 25(3), 519-532.

Brasil. (2014). Implantação das Redes de Atenção à Saúde e outras estratégias da SAS / Ministério da Saúde. Ministério da Saúde.

Bermudez, K. M. \& Batista, R. S. (2017). "Um monte de buracos amarrados com barbantes": o conceito de redes para os profissionais da saúde mental. Saúde e Sociedade, 26(4), 904-919, 2017.

Cardoso, M. R. O. et al. (2017). O mix público e privado no sistema de saúde brasileiro: coexistência em evidência. Saúde em Redes, $3(2)$, 107-118.

Costa, P. H. A. et al. (2015). Sistema de referência e de contrarreferência na rede de atenção aos usuários de drogas: contribuições da análise de redes sociais. Cadernos de Saúde Coletiva, 23(3), 245-252.

Deleuze, G. (2012). Espinosa: filosofia prática. Editora Escuta.

Fusch, P. I. \& Ness, L. R. (2015). Are We There Yet? Data Saturation in Qualitative Research. The Qualitative Report, 20(9), $1408-1416$.

Gil, A. C. (2017). Como classificar as pesquisas? In A. C. Gil. Como elaborar projetos de pesquisa (6th ed.) 25-44. Atlas.

Hoppe, A. S. et al. (2017). Participação popular no Sistema Único de Saúde: olhar de usuários de serviços de saúde. Cinergis, 18(Supl.1), 335-343.

Kantorski, L. P. et al. (2019). Atenção psicossocial infantojuvenil: interfaces com a rede de saúde pelo sistema de referência e contrarreferência. Texto \& Contexto Enfermagem, 26(3), e1890014.

Maximino, V. S. et al. (2017). Profissionais como produtores de redes: tramas e conexões no cuidado em saúde. Saúde e Sociedade, $26(2)$, 435-447.

Meneses, C. S. et al. (2017). O agir leigo e a produção de mapas de cuidado mistos público-privados. Ciência \& Saúde Coletiva, $22(6), 2013-2024$.

Merhy, E. E. (2012). Saúde e Direitos: tensões de um SUS em disputa, molecularidades. Saúde e Sociedade, 21( 2), $267-279$.

Merhy, E. E. et al. (2014). Redes Vivas: multiplicidades girando as existências, sinais da rua. Implicações para a produção do cuidado e a produção do conhecimento em saúde. Divulgação em Saúde para debate, 52, 153-164.

Minayo, M. C. S., Deslandes, S. F. \& Gomes, R. (2016). Pesquisa Social: teoria, método e criatividade. Editora Vozes.

Peduzzi et al. (2020). Trabalho em equipe: uma revisita ao conceito e a seus desdobramentos no trabalho interprofissional. Trabalho, educação e Saúde, 18(Supl. 1), e0024678.

Quintana, R. A. C. et al. (2020). Production of Interprofessional Care for the Person with Oncological Disease: A Study on the Patient's Perspective. Biomedical Journal of Scientific \& Technical Research, 29(3), 22413-22418.

Rocha, K. B. et al. (2016). Transversalizando a rede: o matriciamento na descentralização do aconselhamento e teste rápido para HIV, sífilis e hepatites. Divulgação em Saúde para debate, 40(109), 22-33. 
Research, Society and Development, v. 10, n. 14, e114101421710, 2021

(CC BY 4.0) | ISSN 2525-3409 | DOI: http://dx.doi.org/10.33448/rsd-v10i14.21710

Rosa, N. S. F. et al. (2016). Entre a chave e o cadeado: dobras sobre acesso e barreira na atenção à saúde da pessoa com câncer. In L. M. C. Feuerwerker, D. C. Bertussi, \& E. E. Merhy. Avaliação compartilhada do cuidado em saúde: surpreendendo o instituído nas redes - Livro 2 (1st ed., pp. 92-101). Hexis.

Rosa, N. S. F. et al. (2016b). O SUS-vivo do cotidiano das pessoas: as tensões entre o público e o privado na produção do cuidado em saúde na Bahia. In MERHY, E. E. et al. Avaliação compartilhada do cuidado em saúde: surpreendendo o instituído nas redes - Livro 1 (1st ed., pp. 342-246). Hexis.

Viana, A. L. D. et al. (2018). Regionalização e redes de saúde. Ciência \& Saúde Coletiva, 23(6), 1791-1798.

Seixas, C. T. et al. (2019). O vínculo como potência para a produção do cuidado em Saúde: o que usuários-guia nos ensinam. Interface - Comunicação, saúde e educação, 23, e170627.

Silva, A., Castro-Silva, C. R. \& Moura, L. (2018). Pesquisa qualitativa em saúde: percursos e percalços da formação para pesquisadores iniciantes. Saúde e Sociedade, 27(2), 632-645.

Souza et al. (2017). Produção do cuidado e a rede de atenção a pessoa com doença respiratória crônica: um estudo de revisão. Revista pesquisa em Fisioterapia, 7(4), 127-135.

Tonetto, L. M., Brust-Renck, P. G. \& Stein, L. M. Perspectivas metodológicas na pesquisa sobre o comportamento do consumidor. Psicologia: Ciência e Profissão., 34(1), 180-195. 